\title{
Optimization Approach for Image Forming in Ultrasound Transmission Tomography (UTT): Real Data Case
}

\author{
Tomasz Rymarczyk (iD) ${ }^{1,2}$ and Jan Sikora ${ }^{1,2}$ \\ ${ }^{1}$ University of Economics and Innovation in Lublin, Projektowa 4, 20-209 Lublin, Poland \\ ${ }^{2}$ Research and Development Centre Netrix S.A., Zwiazkowa 26, 20-148 Lublin, Poland \\ Correspondence should be addressed to Tomasz Rymarczyk; tomasz@rymarczyk.com
}

Received 30 November 2018; Revised 5 March 2019; Accepted 8 April 2019; Published 28 April 2019

Academic Editor: Yann Favennec

Copyright (C) 2019 Tomasz Rymarczyk and Jan Sikora. This is an open access article distributed under the Creative Commons Attribution License, which permits unrestricted use, distribution, and reproduction in any medium, provided the original work is properly cited.

\begin{abstract}
Effective image reconstruction algorithm that could be used in Ultrasound Transmission Tomography (UTT) is presented in this paper. The reconstruction process was bringing over to the solution of the inverse problem which was converted to the optimization task. The optimization parameters were selected as follows: a position vector defining the position of the obstacle centre (the length of the position vector and its angle) and the internal radius of the test objects (obstacles). In this case, sixteen trial objects were selected, which gives 48 th optimization parameters (three parameters for each trial object). During the optimization process some of them could diminish to zero; the others would change the position and dimensions in such a way that the calculated signal become as close as possible to the measured one. The objective function and linear inequality constrain were defined. The optimization process is very sensitive how far away from the optimal solution the starting point was defined. That is why three cases of the selection of the starting point to the optimization process were considered.
\end{abstract}

\section{Introduction}

The idea of optimization approach, among the others [18], is very popular for the inverse problem's solution [9-14] and could be successfully applied in ultrasonic image reconstruction. This is not a new idea $[2,9,15,16]$ and as a matter of fact is very simple. However, the difficulties as usually are hidden in detail. Those difficulties understand as, for example, implicit errors will be discussed in subsequent paragraphs of the paper.

The paper concentrates on reconstruction algorithm based on real measurements provided by Netrix R\&D Company. The NETRIX tomograph is working in transmission mode, so reflection signals are not measured. In this work all, simplifying assumption was introduced along with the fact that ultrasound wave is propagated along strait lines [16]. Despite such strong simplifying assumptions resulting images are satisfactory with respect to the imaging precision and could be applied in the industry.
The method of optimizing the solution to the inverse problems is very sensitive to the choice of the starting point. Therefore, this article presents three different cases. In the first case, due to the analysis of the measured signal, it was shown how to not only determine the number of internal objects, but also relatively precisely determine their location.

In the second case, to determine the starting point, a hybrid approach was presented using the ART method. The ART approach makes it possible to cover the existence of a possible object, which would then be subject to optimization. However, both approaches for defining the starting point and those mentioned above have certain disadvantages.

Therefore, a third approach to the optimization process was proposed. This third approach was called a generalized starting point. This approach has already been successfully used in nonclassical problems (not X-ray tomography). The solution to the problem starts with several (in this case sixteen) test objects, randomly or rather with a certain algorithm, located in the studied region. The generalized 


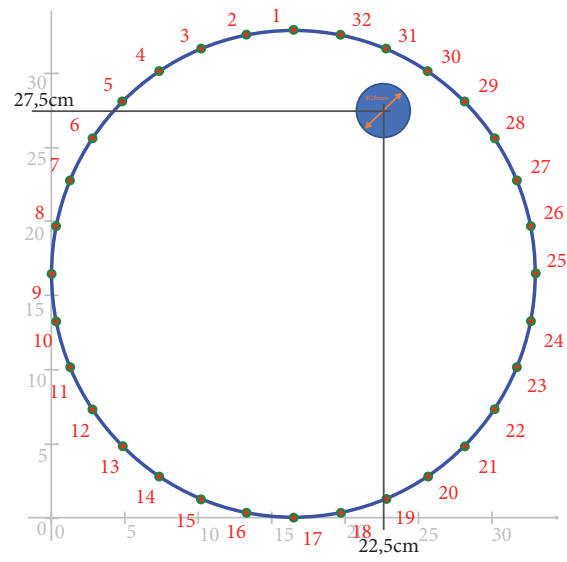

(a)

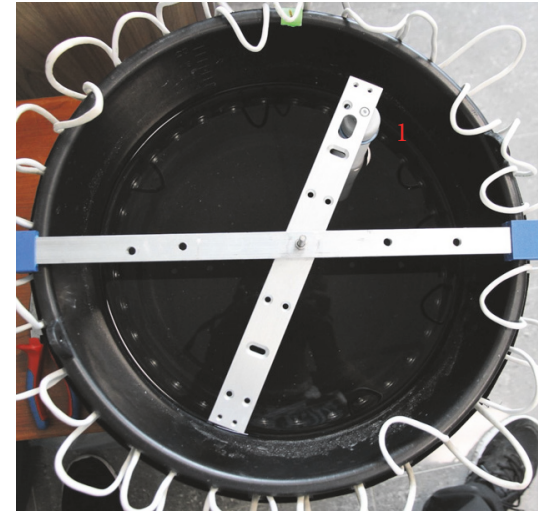

(b)

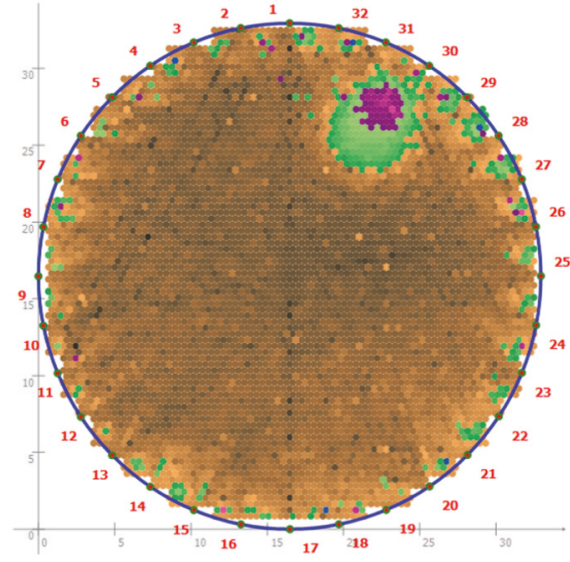

(c)

FiguRE 1: Region under investigation. (a) Internal object position. (b) General view of the laboratory stands. (c) Results of reconstruction with the aid of advanced methods of measurements conditioning and filtration [17].

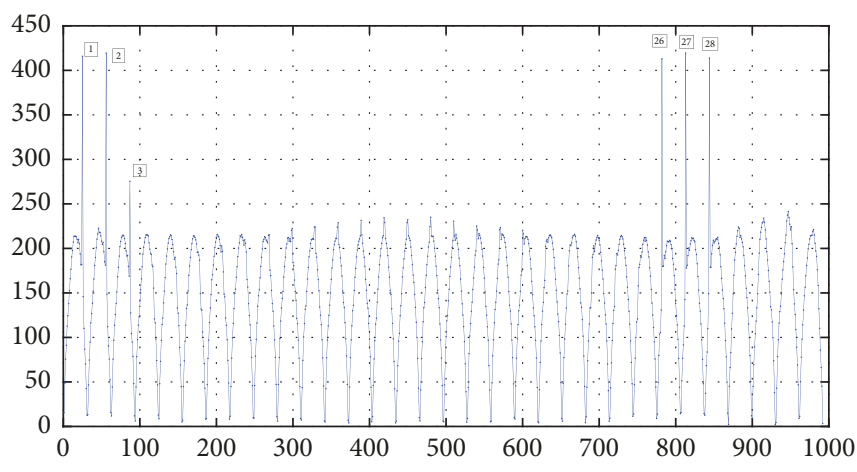

(a)

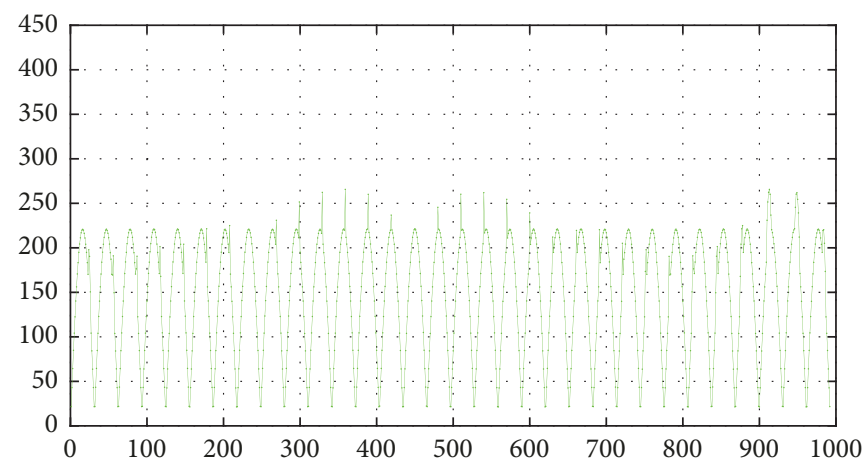

(b)

Figure 2: Measured signal. (a) In the laboratory stand with marked numbers of the projection angles. (b) Signal accomplished after optimization iterative process.

starting point has only one drawback, namely, a large number of optimization parameters.

This article has proven that Ultrasonic Transmission Tomography (UTT) for industrial processes is able to offer reliable images.

\section{One Internal Object Case}

At the beginning let us consider relatively simple problem of identification of identifying the dimensions and location of a single object inside a water-filled tank. As it is shown in Figure 1(a) the cross section of the tank is circular with the radius equal to $330 \mathrm{~mm}$.

It seems that such a problem seems easy, but difficulties are hidden in laboratory measurement data. The only conditionality of measurement data passed during averaging of five measurement frames was adopted. The results obtained will be presented later in this work.

There are many reconstruction techniques $[15,18-24]$ but in this paper, as a traditional reconstruction method we will understand the reconstruction with the aid of Algebraic Reconstruction Technique (ART), method which was extensively discussed for example in [5]. ART method allows achieving the reliable images based on laboratory measurements [4]. The measured signals from the laboratory stand shown in Figure 1(b) are presented in Figure 2(a).

In Figure 2 one can observe how the transmissions delay times of acoustic wave sharply increase if the obstacle (the plastic bottle fulfilled with the air presented in Figure 1(b)) occurs in the path of the beam. The tank was fulfilled with the water. The speed of the sound in water is $1481 \mathrm{~m} / \mathrm{s}$ [25] but for air (bottle) only $343 \mathrm{~m} / \mathrm{s}$ [26]. Of course, the speed of sound depends on many different parameters such as, for example, temperature, but for normal conditions (room temperature about $20^{\circ} \mathrm{C}$ ) such parameters could be assumed as mentioned above [26].

As can be seen in Figure 2(a), the image of the measurement signal is regular and has some symmetry features. Figure 2(b) shows the resultant signal obtained after the optimization process, when the objective function decreases to almost zero.

As already mentioned, the only condition of the measurements was that an average value was calculated from the fifth measurement frame. The reconstruction was based on those 


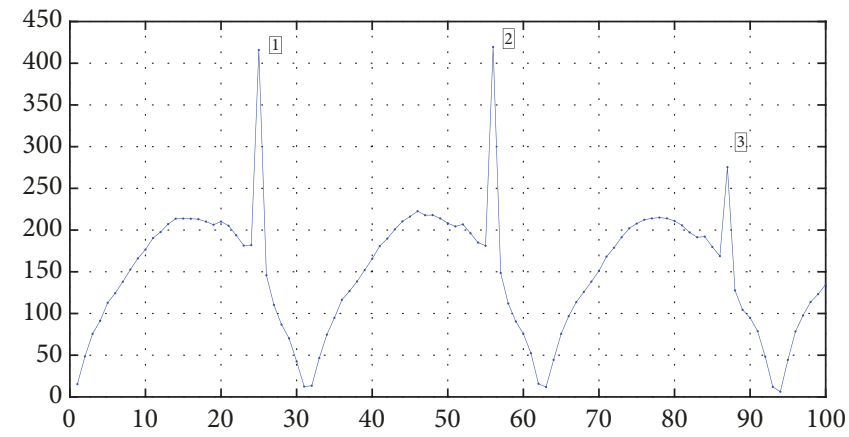

FiguRE 3: Enlarged measured signal for selected projection angles.

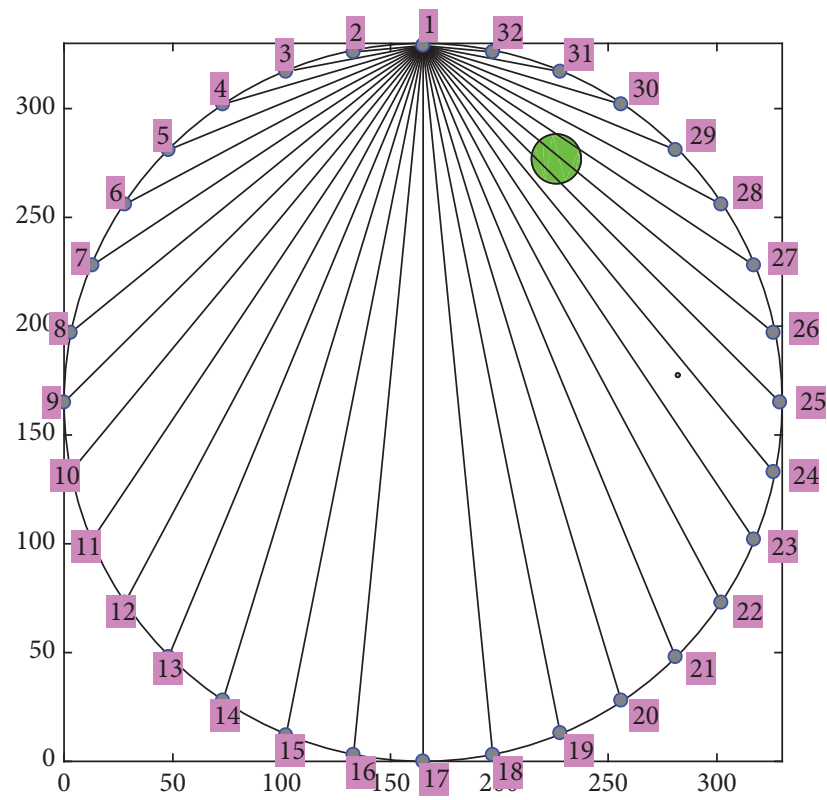

(a)

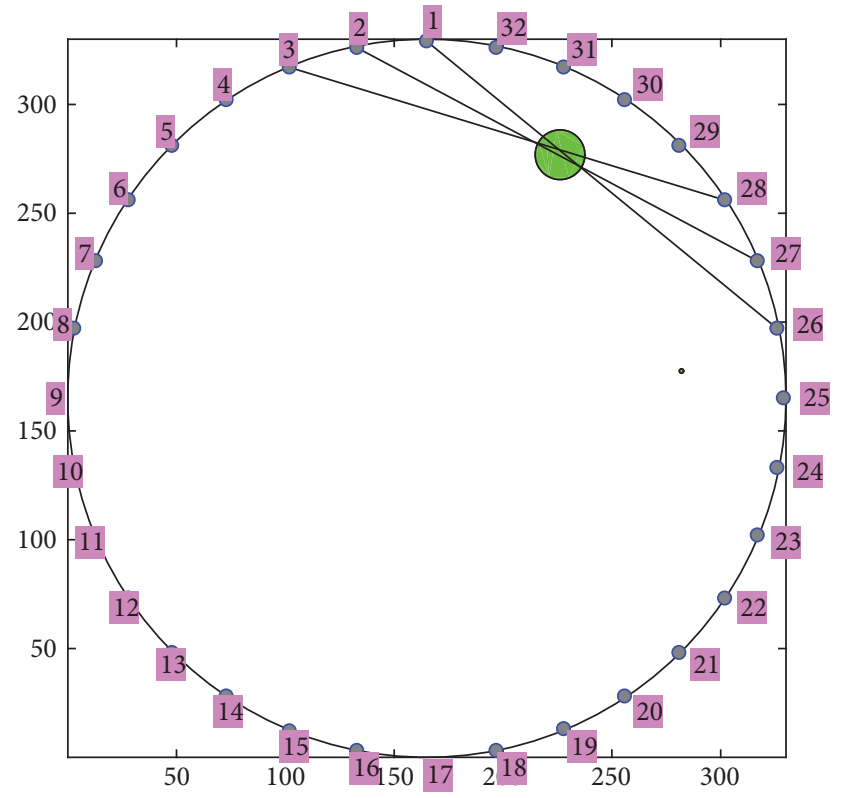

(b)

Figure 4: One object position inside the region. (a) Sensors location and distribution of rays for the first projection angle. (b) Three rays corresponding to the three peaks for the first three projection angles shown in Figure 3 and also for the 26-th, 27-th, and 28-th projection angle.

values. For the 1, 2, and 3 and 26, 27, and 28 projection angles (see Figure 2), the picks in the measured signal are clearly visible. The picks are caused by crossing the acoustic wave through internal object fulfilled with a material with a lower velocity than the velocity of the background (water). For the first three projection angles the enlarged and normalized measured signal is presented in Figure 3.

So, the total time which signal needs to travel from the transmitter to the receiver became longer. This could be clearly seen in Figures 2 and 3 as a single peak for the certain projection angles. In this case that was the first second and the third projection angle (see, for example, Figure 3 ) as well as for the 26-th, 27-th, and 28-th projection angle as it could be seen in Figure 4(b).

As the projection angles are known, it is easy to calculate (considering the measuring points denoted as the dots in Figure 3) to which of the sensor's ray is moving. If those rays will be drawn, then one gets the set of straight lines crossing in a certain region. In such a way the place of location of the single object could be indicated as it is presented in Figure 4(b).

Due to this procedure for the optimization approach, with relatively high precision the location of the single object could be indicated as the place of three rays crossing (see Figure 4(b)). The method which defines the starting point by analysing the measurement signal can meet with the accusation of high labour consumption. First the measured signal must be analysed (not always it should be so easy without of clearly visible peaks as it is present in Figure 2) and then the position of the internal object should be proposed on this basis. Additionally, the shape of the imaged object should be known. In this case the cross section of internal object is a circle. For the inverse problem's solution, engagement of additional knowledge is a common practice [27].

Unfortunately, such an approach possesses one big disadvantage. It is very difficult to automate the reconstruction 


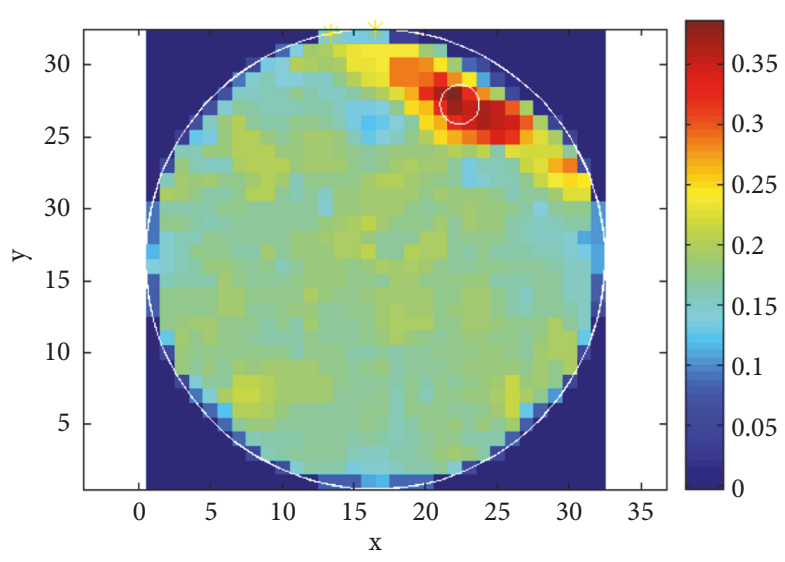

(a)

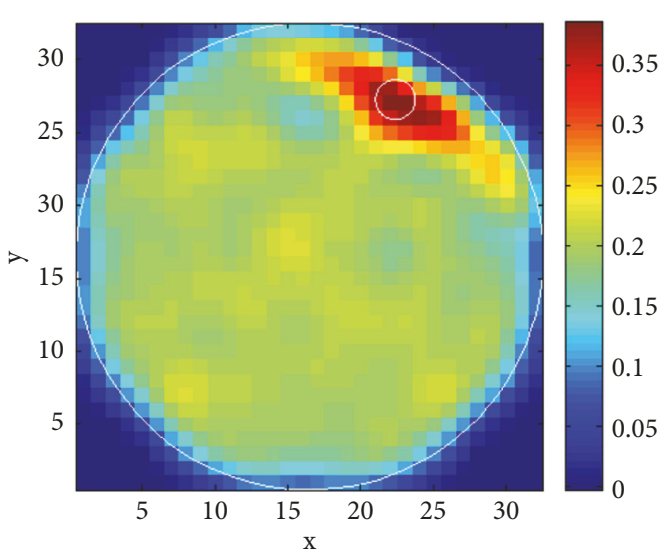

(b)

FIGURE 5: Images for the case illustrated in Figure 1 without of measured signal conditioning. (a) After treatment by median filter (built in function of MATLAB). (b) After the Mexican Hat filtering.

process. In some applications it could disqualify such an approach. Therefore, a hybrid approach is suggested.

What does it mean the hybrid approach in this context? By the hybrid approach the authors understand the introductory reconstruction with the aid of the ART method. Such an image helps to define the starting point to the optimization process as it is seen in Figures 5(a) and 5(b). For the single object the problem is simple and obvious. The problem becomes more difficult for more internal objects. Then the hybrid approach would be very helpful and could indicate the subregion where eventually the sought objects could be present.

Hybrid approach would not have above-mentioned disadvantage and in the same time would keep all the advantages of the previous approach.

The image achieved by ART method is presented in Figure 5. In this figure by white colour circle the real position and dimension of the inner object are denoted. As could be seen the position of the object is correct, but the image is blurred so it is difficult precisely describe its position and dimension.

In example presented in Figure 5(b), one can observe the effectiveness of Mexican Hat filtering in comparison to the simplest median filter [28]. The background of the region of interest is more regular than in Figure 5(a). As before the measured data were not conditioned.

In order to improve the image presented in Figure 5, the authors proposed the optimization approach. The ART method shows the possible location of the internal obstacle, but knowledge of the problem provides its circular shape. Thus, the optimization approach can provide a more precise image due to additional knowledge about the shape of the object (obstacle) to be imaged.

As a starting point, the two objects simulated the blurring effect in Figure 5, with different radiuses selected, as shown in Figure 6. Those two starting objects are placed in the location pointed out by the ART method.
In order to simplify the optimization problem, the parameters of candidate obstacles were defined in polar coordinates because the considered region and obstacles are circular. As a result, inequalities are linear. For Cartesian coordinates, the limitations of inequality would become nonlinear, always more difficult to handle.

As the optimization tool the fmincon MATLAB build in function was selected [28].

Initially, it is not always possible to determine the location of the internal object in the considered region. Than the third approach looks the most convenient way to solve such problem. It consists on the assumption that the object which is sought could be everywhere inside the region. So, in such case, knowing that the cross section of the internal object should be a circle, sixteen candidate circular objects are distributed inside the region under consideration as it is shown in Figure 7.

The candidate internal objects during the optimization process could change their radiuses, position vectors and the angles of the position vectors (parameters of optimization process, design parameters) in a similar way as it was proposed in [9].

\section{The Objective Function Definition}

In order to fit calculated in each iteration step signal to the measured signal, the following objective function has been defined:

$$
\begin{aligned}
\Phi & =\sum_{j=1}^{j=p} \Phi_{j}=\frac{1}{2} \sum_{j=1}^{j=p}\left(\mathbf{f}_{j}-\mathbf{v}_{0 j}\right)^{T}\left(\mathbf{f}_{j}-\mathbf{v}_{0 j}\right) \\
& =\frac{1}{2}\left(\mathbf{F}-\mathbf{V}_{0}\right)^{T}\left(\mathbf{F}-\mathbf{V}_{0}\right),
\end{aligned}
$$

where $\Phi$ is the total value of objective function calculated for all $p=32$ projection angles (positions of the sound sources), 


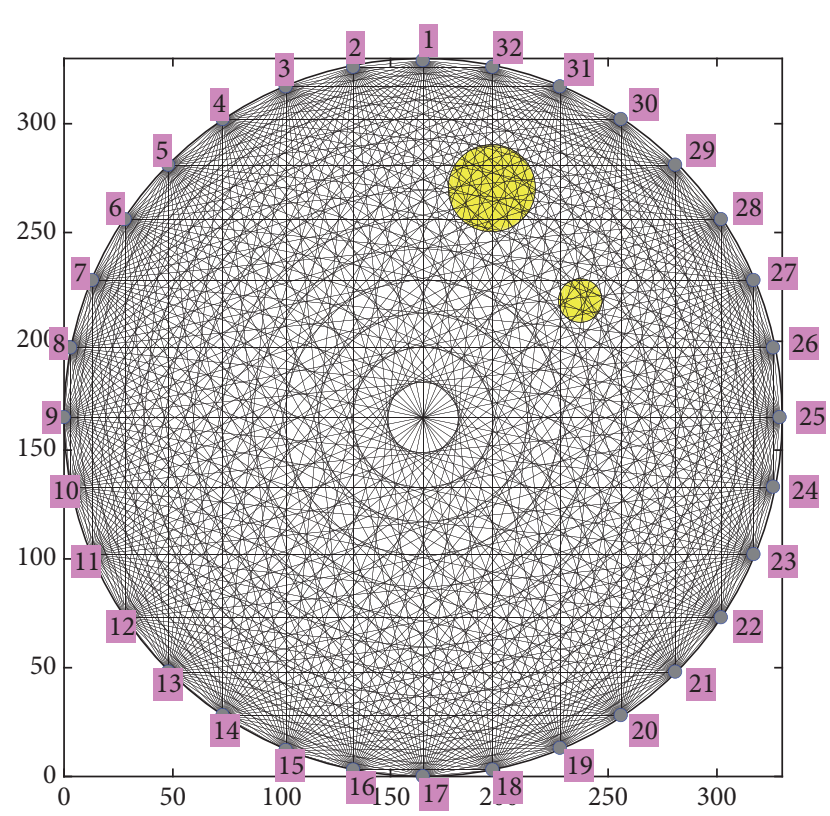

(a)

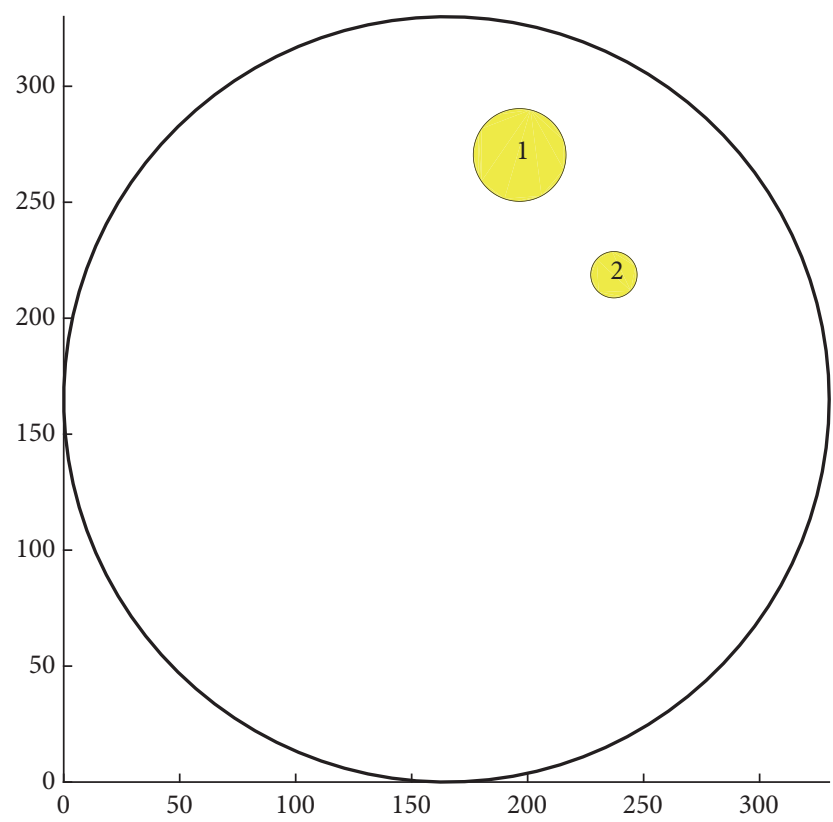

(b)

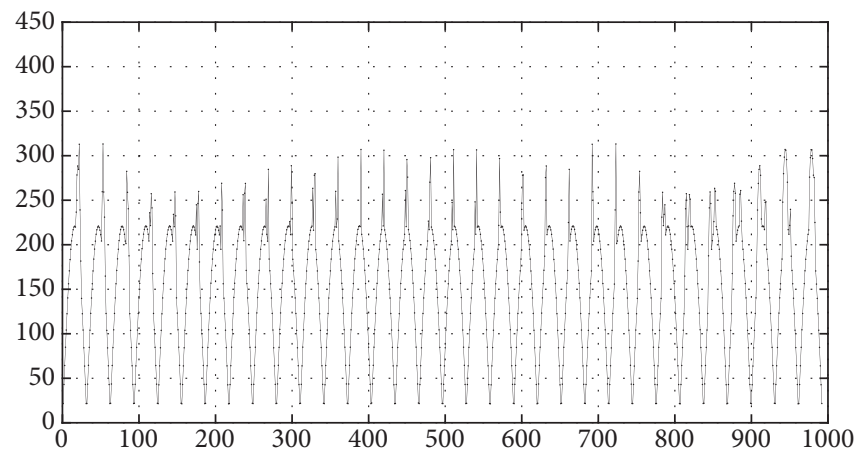

(c)

FIGURE 6: The starting point. (a) With the ray's distribution inside the region. (b) Numbers of the candidate objects as a starting point. (c) "Measured" signal corresponding to the starting point.

$j=1,2, \ldots, p, \Phi_{j}$ is objective function for $j$-th position of the sound source, $\mathbf{f}_{j}$ is vector (one column matrix) of a sound wave transition times calculated in certain iteration step for assumed distribution of internal objects for the $j$ th projection angle, $\mathbf{v}_{0 j}$ is measured vector of times in $\mu s$ for the $j$-th projection angle, $\mathbf{F}=\left[\mathbf{f}_{1}, \mathbf{f}_{2}, \ldots, \mathbf{f}_{p}\right]^{T}$, and $\mathbf{V}_{0}=$ $\left[\mathbf{v}_{01}, \mathbf{v}_{02}, \ldots, \mathbf{v}_{0 p}\right]^{T}$.

The objective function $\Phi_{j}$ will be minimized taking into account given linear inequality constraints. $\Phi_{j}$ is Mean Squared Error (MSE) of image forming for $j$-th projection angle. The value of the total objective function for the given measuring data depends on matrix $\mathbf{F}$. In turn, the matrix $\mathbf{F}$ is not only the function of internal object parameters like the object radius and position vector as well as positions vector angle (in degrees) measured from positive direction of $\mathrm{x}$ axis, but also the function of projection angle. The matrix $\mathbf{F}$ is defined in each iteration step by the solution of the forward problem.

\section{The Linear Inequality Constrains}

For the generalized starting point (see Figure 7) there are 16 internal candidate objects. Each possesses three parameters defining their position and dimension. So, the total number of decision parameters (optimization parameters) is equal to $16 * 3=48$. For those 48 decision parameters, the limitations of inequality should be imposed.

Let us assume that the radius of the cross section of the object has to be a positive number along with the length of its position vector. That already completes 32 inequality constrains. Additionally, it could be assumed, however it is not necessary, that the angle of the position vector is positive as well. That increases the number of inequality constraints up to 48 .

The starting point shown in Figure 7 is not the only one. But for such starting point some protection against overlapping candidate internal objects must be undertaken. The 


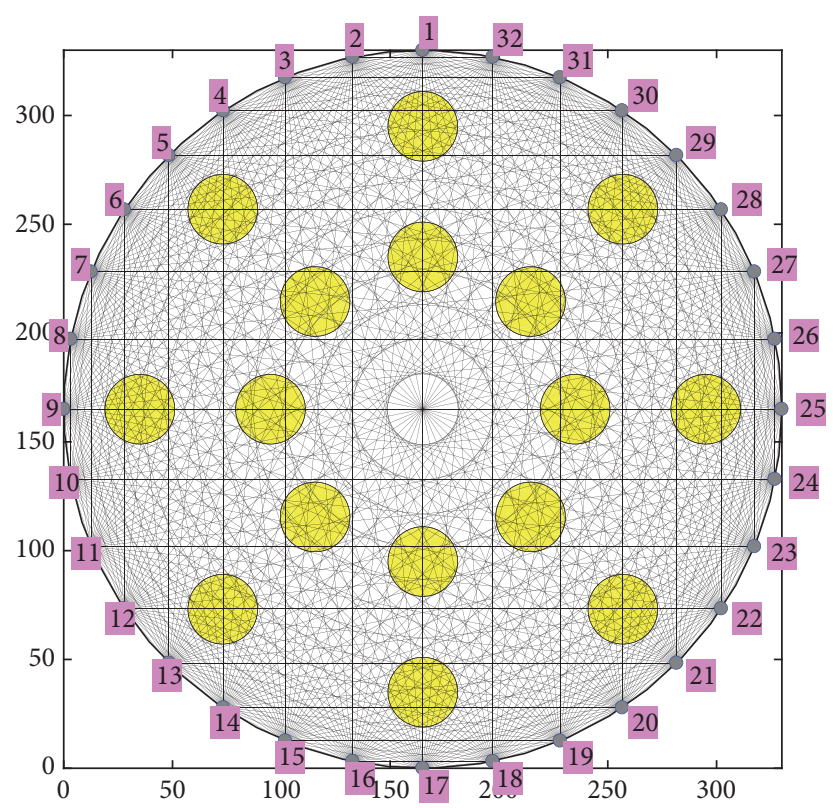

(a)

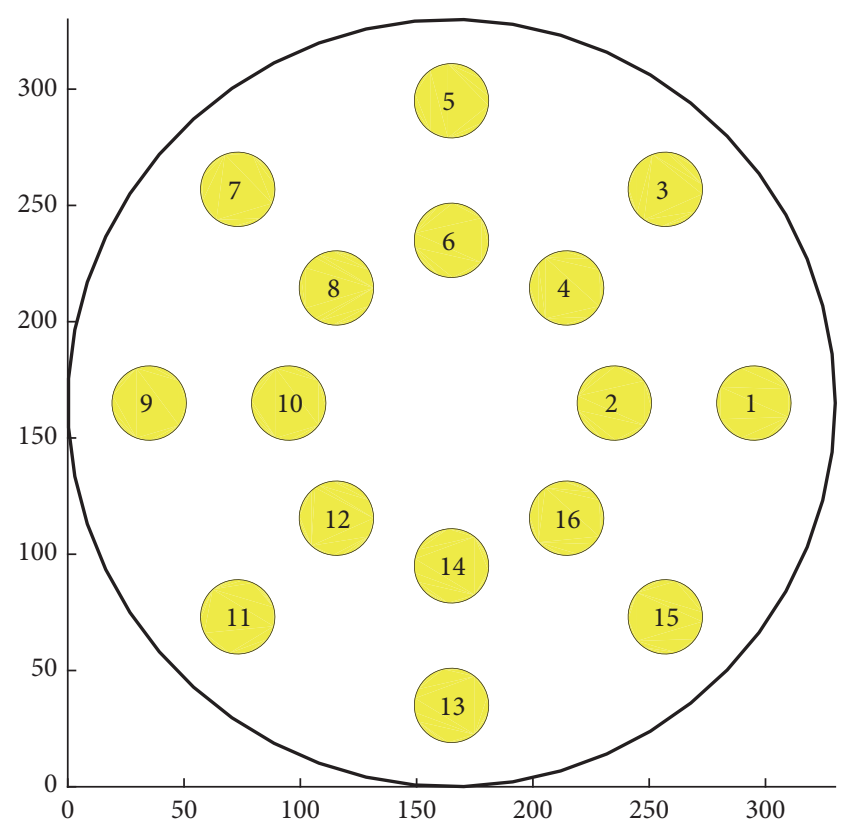

(b)

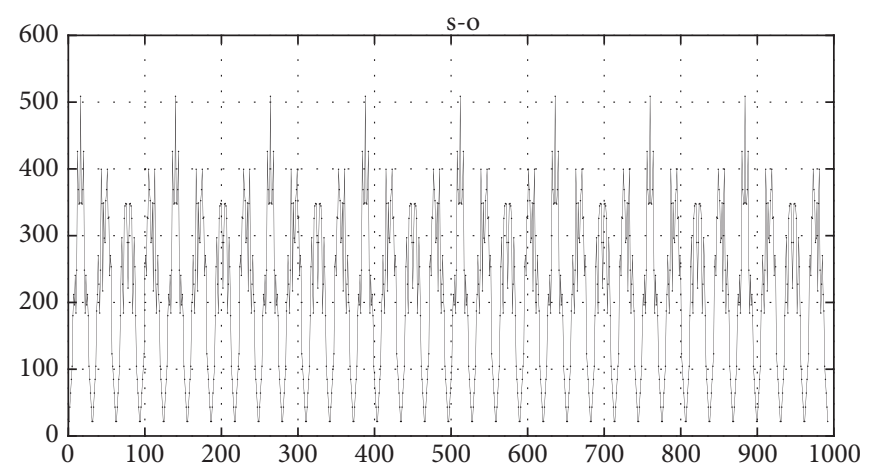

(c)

FiguRE 7: Generalized starting point. (a) Internal objects with the ray's distribution. (b) Numbers of candidate objects. (c) "Measured” signal calculated for the starting point.

objects overlapping could happen when they would be moving during the iteration process. The algorithm in present form is not able to take such situation into account. The inequality constrains protecting against objects overlapping will take the following form:

$$
R_{i}-r_{i}-\left(R_{i+1}+r_{i+1}\right)>R_{w} * 0.01,
$$

where $R_{i}$ is the length of the position vector for $i$-th internal object and $r_{i}$ is the radius of internal objects cross section (see Figure 7).

Finally, the inequality matrix will possess 64 rows and 48 columns.

\section{Results of Reconstruction}

This problem was solved three times. In every case the starting point for optimization process was selected using different approach, firstly, by analysing the measured signal, secondly with the aid of the ART method (called the hybrid approach), and last but not least using the so-called generalized starting point shown in Figure 7(b).

For the case when the starting point was determined by analysis of measured signal and for the hybrid approach (see Figure 6) the results of reconstruction are presented in Figure 8. The results of reconstruction are almost identical for the first two starting points and are in good relation to real position and dimension of the object marked by the black circle (Figure 8(b)).

The resulting object marked in green colour is a little bit smaller and its centre is placed slightly higher than the real object. The iterative process as it could be seen in Figure 8(c) was stable and objective function was reduced several times. For the hybrid approach the radius of one of the two objects defining the starting point was reduced nearly to zero, but the other was placed in the expecting position as it is seen in Figure 8(a) or Figure 8(b).

The measured signal is presented in Figure 2(a), and for comparison the signal which was achieved from optimization 


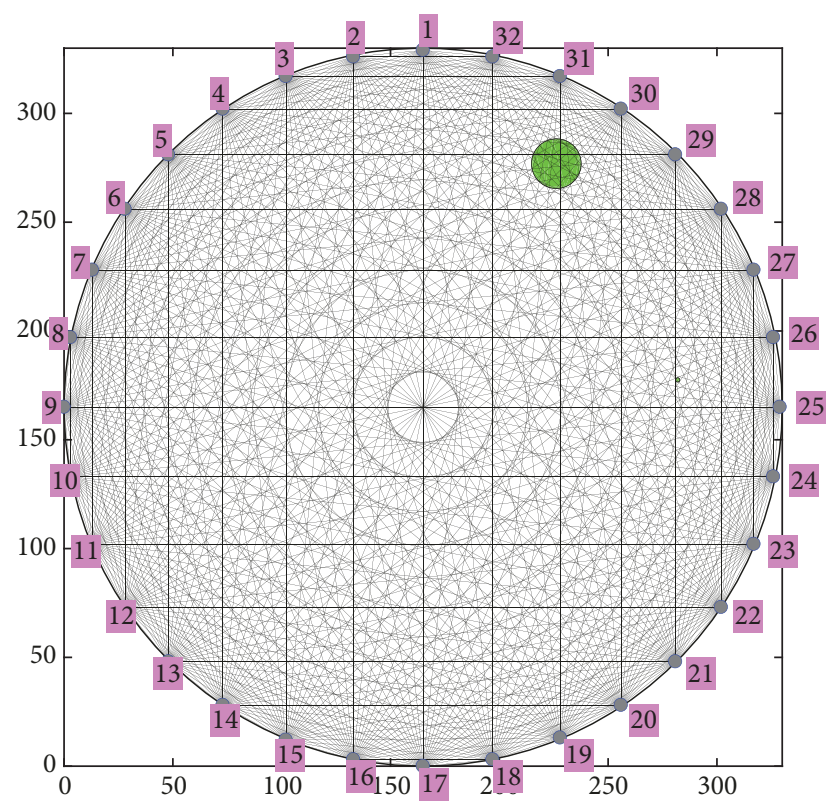

(a)

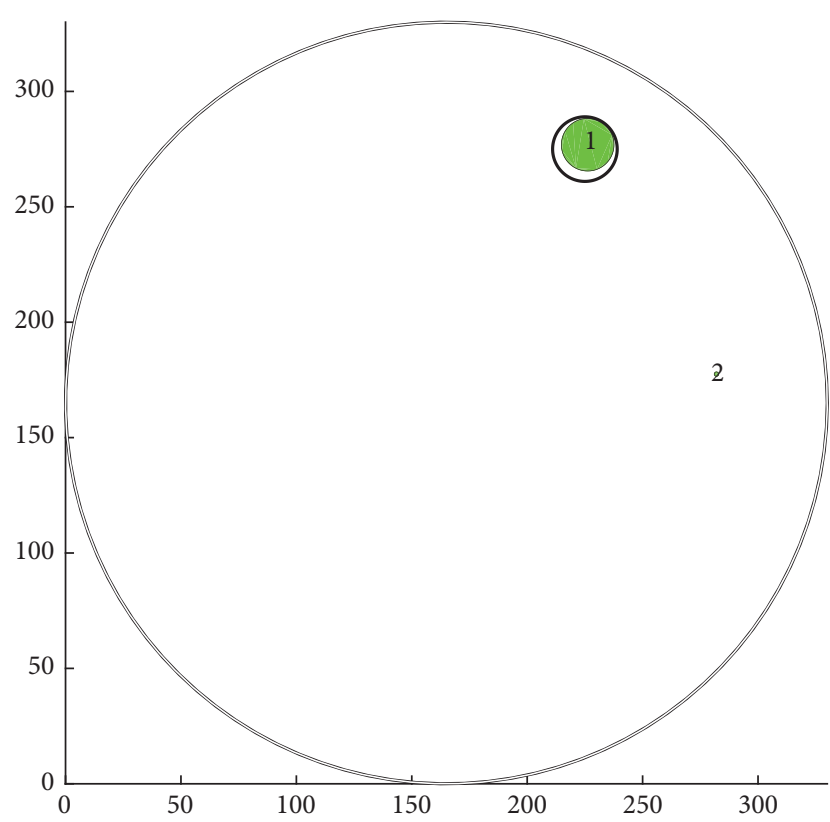

(b)

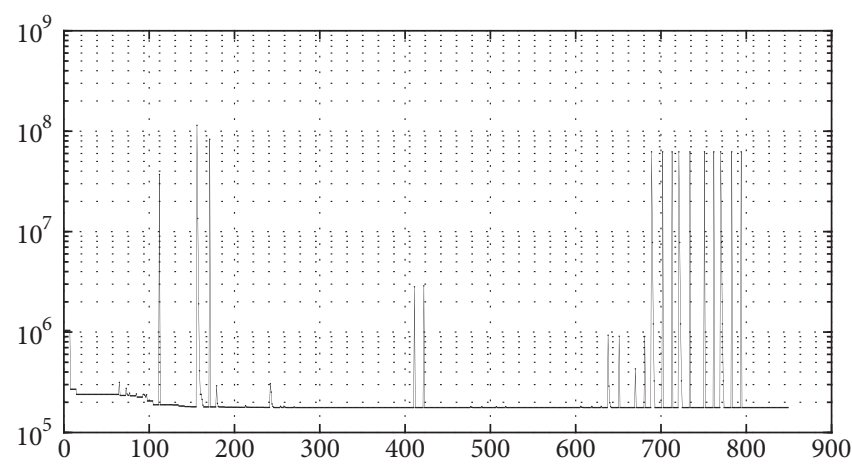

(c)

FiguRE 8: Results of reconstruction. (a) With the ray's distribution. (b) The real position and dimension of internal object denoted by black circle. (c) The optimization processes.

process is shown in Figure 2(b). As could be noticed those signals do not match perfectly each to the other. Comparing both signals, we must consider the point from which the optimization was started. The starting point generated the signal shown in Figure 7(c). This signal is essentially different than the measured one. Additionally, the colour map of the measured signal array (horizontal axis is the number of projection angles, but the vertical axis is the ray number) could be compared with the colour map of calculated signal array. The similarity of both signal arrays is pretty obvious (see Figure 9).

If the results of the of the optimization process are not satisfactory always the iterative process could be restarted from new modified starting point. Not always such an approach is convenient. It demands operator intervention in optimization process like, for example, selection of internal objects for the restart.

More universal option is application of generalized starting point as it is suggested in Figure 7(b). This is the third approach to the starting point definition of the optimization. Result of reconstruction in this case is presented in Figure 10(b). The numbers of the candidate objects whose radiuses are reduced almost to zero are clearly visible. Only one of the sixteen candidate objects remain (see Figure 10(b)). However, its dimension and position are not satisfactory jet.

Results for the generalized starting point are less precise than in two previous cases. It could be explained by much bigger dimensions of constrained optimization problem. For generalized starting point we have 16 internal objects. Each has three decision parameters. In previous cases it was only six decision parameters instead of 48 in the present case.

As can be seen in Figure 10(b) the background of the image is polluted by 15 very small objects which have no influence on the calculated signal. So, for the new starting point they could be ignored. The restart reformulates the optimization problem reducing the number of decision parameters from 48 up to only three (Figure 11(a)). 


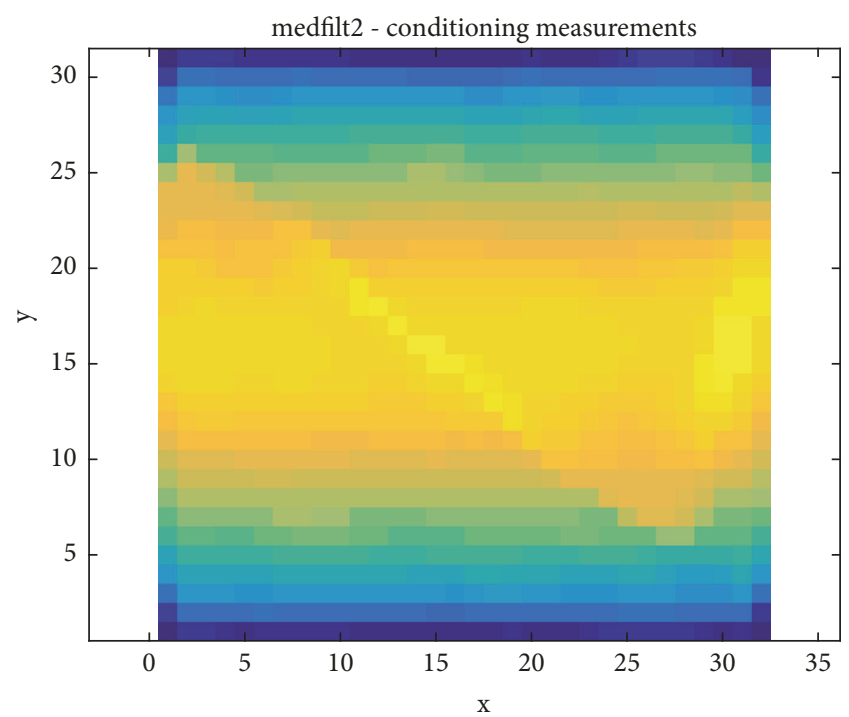

(a)

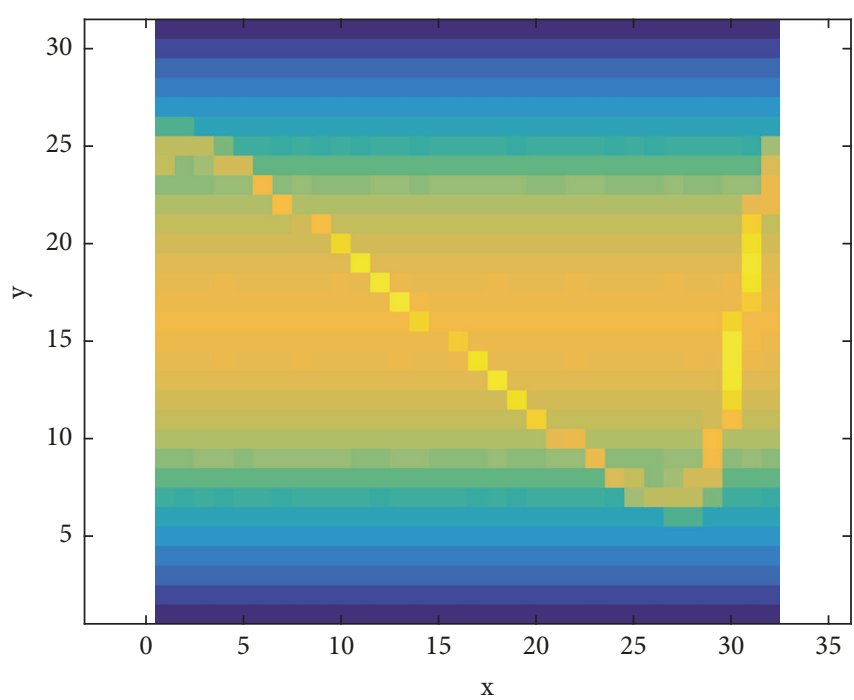

(b)

FIGURE 9: Colour map of the signal matrix. (a) Measured. (b) Calculated during reconstruction process.

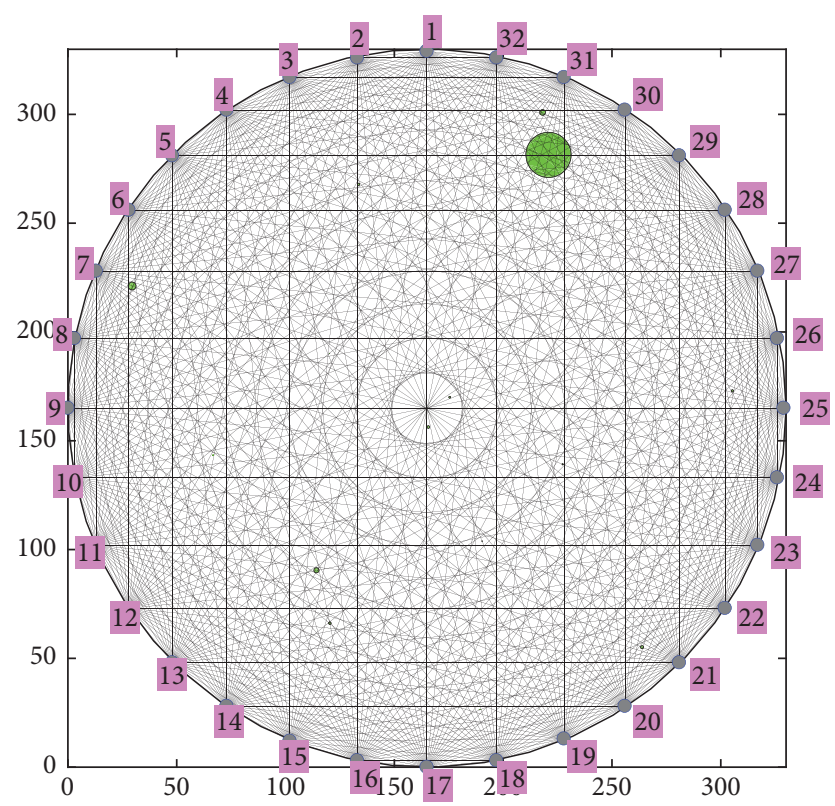

(a)

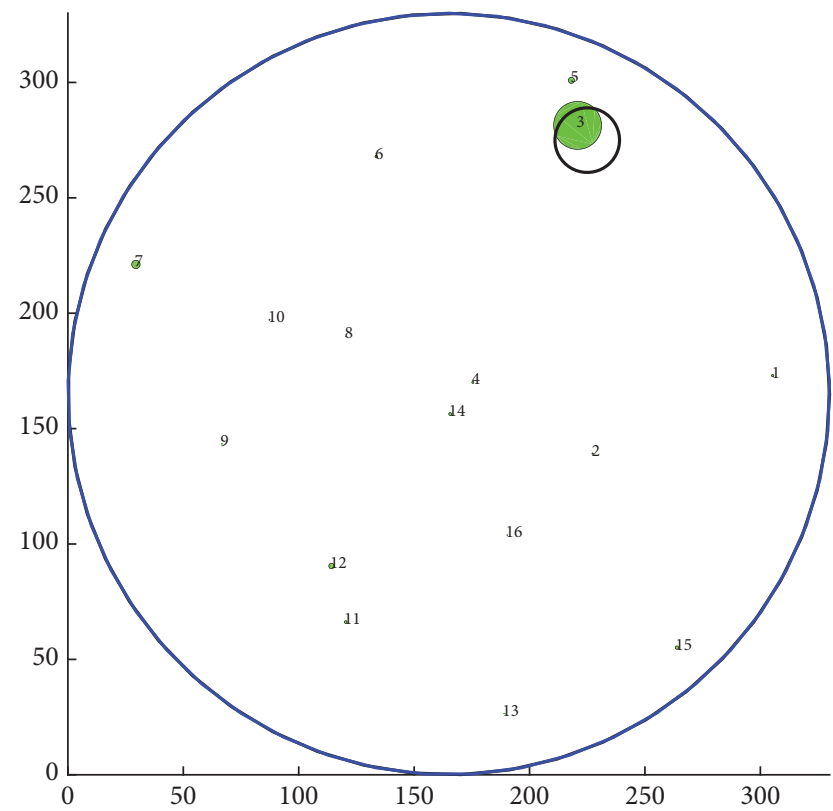

(b)

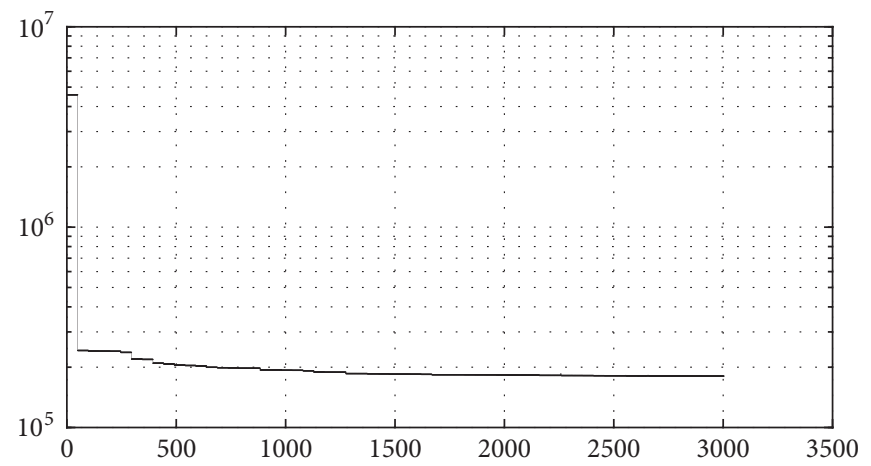

(c)

FIGURE 10: Reconstruction results. (a) With rays and sensors positions. (b) With the real object marked by the black circle. (c) Objective function distribution versus number of iteration steps. 


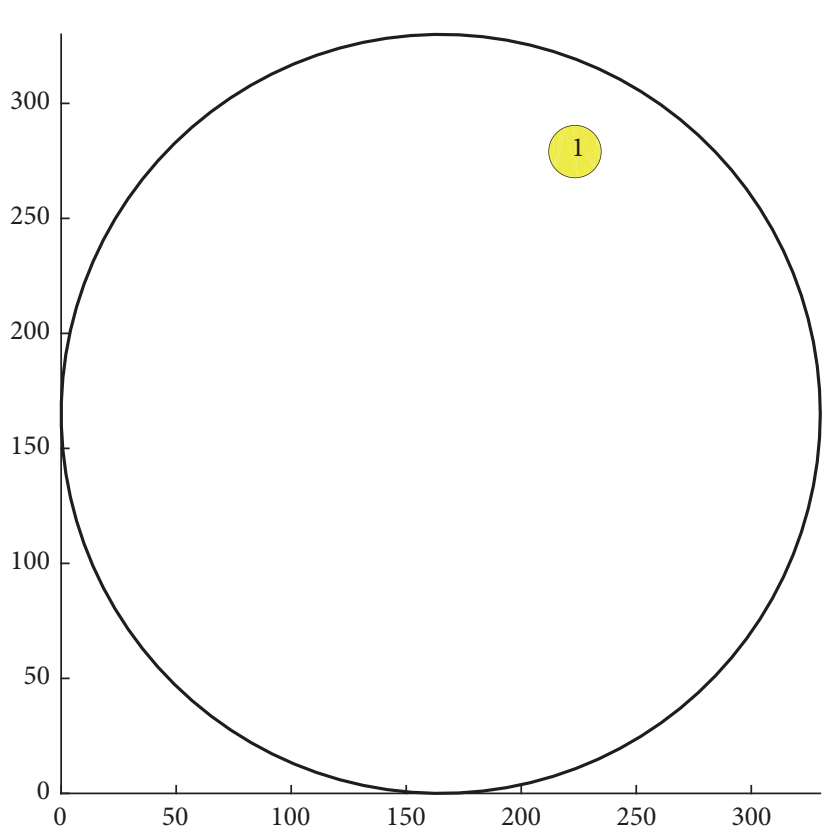

(a)

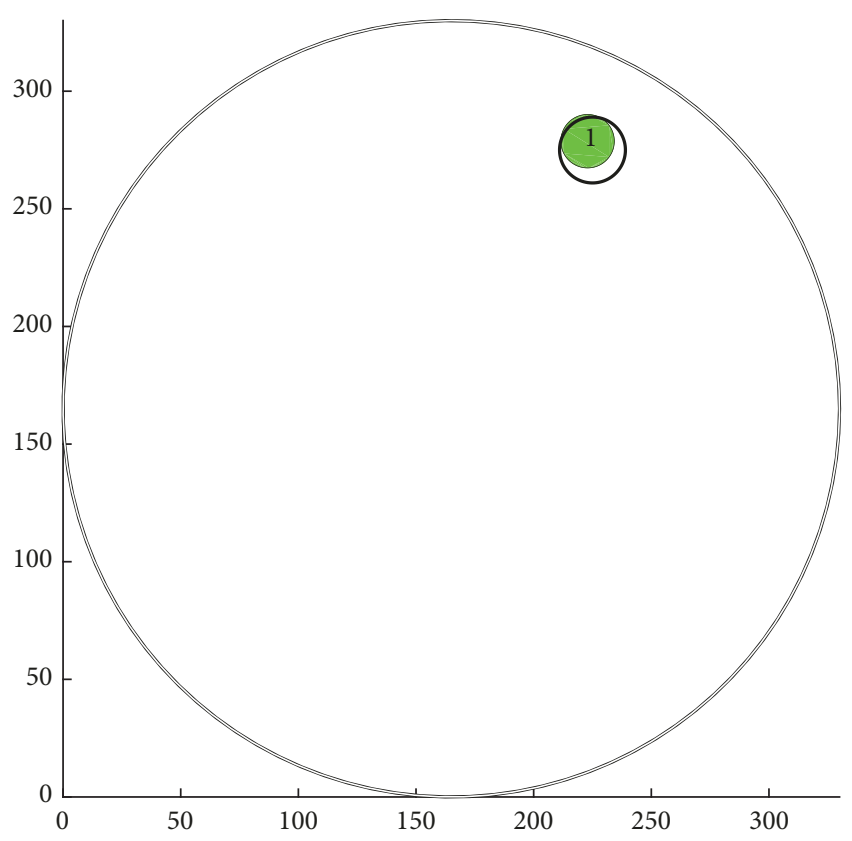

(b)

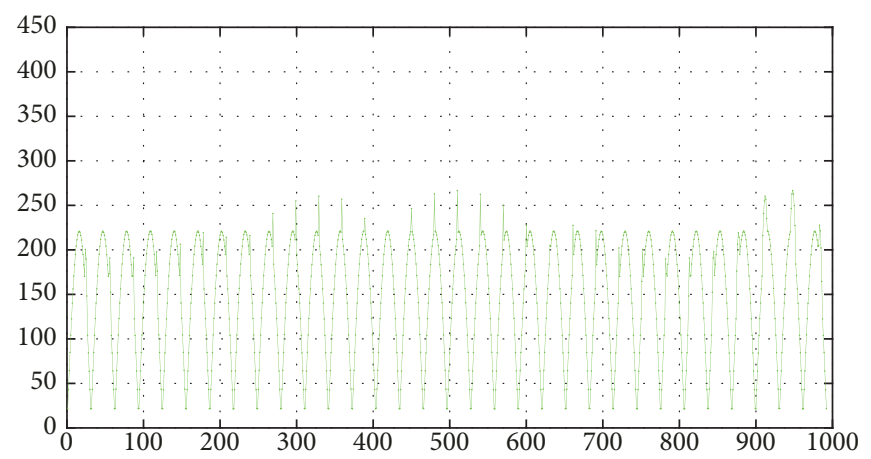

(c)

FIGURE 11: Restart. (a) New starting point without the candidate internal objects with the radiuses close to zero. (b) Image after restart. (c) Signal calculated in the iterative process.

TABLE 1: Basic design parameters for the reconstruction.

\begin{tabular}{lccc}
\hline & Radius [mm] & Length of position vector [mm] & Angle of position vector [deg] \\
\hline Starting point & 9.60 & 130.80 & 65.00 \\
Final result & 11.39 & 128.13 & 62.88 \\
Objects model & 14.00 & 125.30 & 61.40 \\
Relative error & $18.64 \%$ & $2.26 \%$ & $2.41 \%$ \\
\hline
\end{tabular}

After restarting (Figure 11(a)) the results shown in Figure 11(b) have been improved, but they are still slightly worse than in the two previous cases. The final results after restart with the relative error are shown in Table 1.

The radius of the cross section of internal object has the biggest relative error and it does not exceed the $20 \%$.

In Figure 12(a) the colour map of the measured signal matrix is compared to the calculated one, where (b) belongs to the calculation before the restart and (c) after the restart. The image in Figure 12(c) is slightly better and is similar to the measured signal matrix.

\section{Conclusions}

This article presents a new effective reconstruction method in Ultrasonic Transmission Tomography. Comparisons of achieved results by the proposed approach (see, for example, Figure 11(b)) with the reconstruction results offered by the ART method (presented in Figure 5(a) or Figure 5(b)) have to lead to the conclusion that the new approach gives much better image. The fact is that knowledge about the region in question has significantly helped achieve such good results. However, the ART method could be recognized as more 


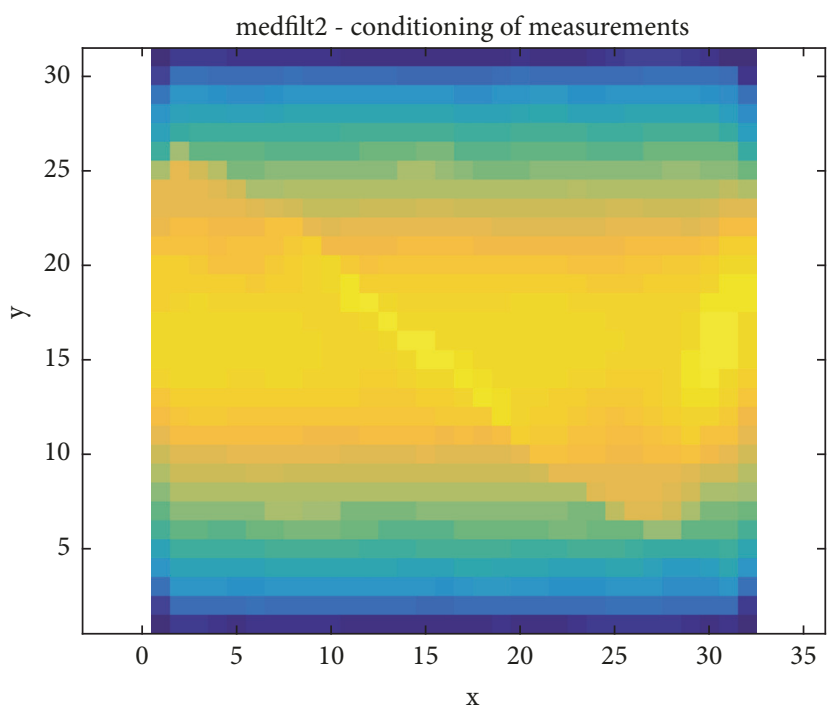

(a)

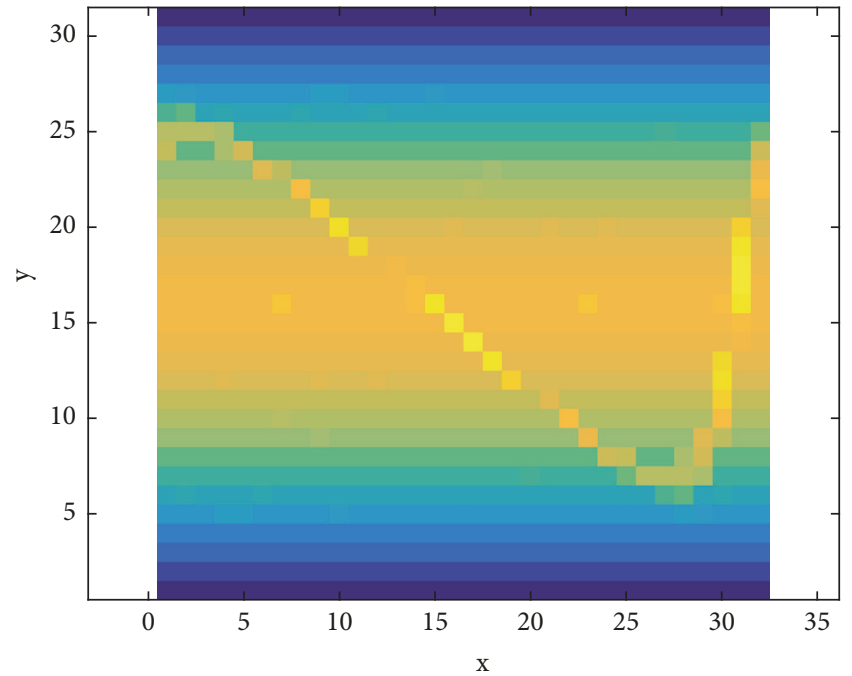

(b)

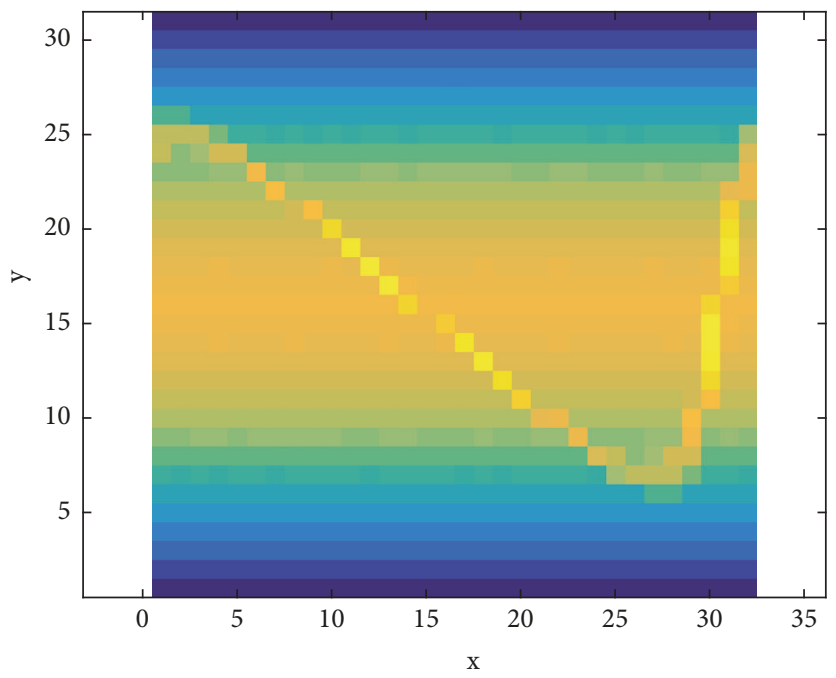

(c)

Figure 12: Colour map of signal matrix. (a) Measured. (b) When the iteration process was stopped for the first-time. (c) After restart of the iteration process.

universal, which do not need any special knowledge. That is why the ART method, what was proved in this paper, could be very useful for determination of the starting point to the optimization procedure.

In general, the optimization approach to the inverse problems is very sensitive on how far the starting point is from the optimal solution. As a criterion of the optimization the global (i.e., for all projection angles) Objective Function representing the Mean Squared Error (MSE) of image forming was selected. It means that in the iterative process the distance between the measured signal and calculated signal in each iteration step is minimized.

This research shows that the proposed algorithm is suitable for reconstruction and is able to provide good quality images as well as the classic ART algorithm. In conclusion, the proposed approach is an effective tool for image reconstruction in Ultrasonic Transmission Tomography. The quality of resulting images is very high not exceeding $20 \%$ of a relative maximal error. It is worth stressing that such result was achieved without any conditioning of measured data.

\section{Data Availability}

Data (algorithms and measurements) can be made available to readers in the form agreed with the publishing house.

\section{Conflicts of Interest}

The authors declare that there are no conflicts of interest regarding the publication of this paper. 


\section{References}

[1] J. Dusek, D. Hladky, and J. Mikulka, "Electrical impedance tomography methods and algorithms processed with a GPU," in Proceedings of the Progress In Electromagnetics Research Symposium - Spring (PIERS), pp. 1710-1714, Russia, 2017.

[2] H. Garbaa, L. Jackowska-Strumiłło, K. Grudzień, and A. Romanowski, "Application of electrical capacitance tomography and artificial neural networks to rapid estimation of cylindrical shape parameters of industrial flow structure," Archives of Electrical Engineering, vol. 65, no. 4, pp. 657-669, 2016.

[3] K. Grudzien, Z. Chaniecki, A. Romanowski, D. Sankowski, J. Nowakowski, and M. Niedostatkiewicz, "Application of twinplane ECT sensor for identification of the internal imperfections inside concrete beams," in Proceedings of the 2016 IEEE International Instrumentation and Measurement Technology Conference, I2MTC 2016, pp. 1-6, Taiwan, 2016.

[4] T. Gudra and K. J. Opielinski, "The multi-element probes for ultrasound transmission tomography," Journal de Physique, vol. 137, pp. 79-86, 2006.

[5] G. T. Herman and W. N. Brouw, Image Reconstruction from Projections: The Fundamentals of Computerized Tomography, Academic Press, New York, NY, USA, 1980.

[6] A. Romanowski, "Contextual processing of electrical capacitance tomography measurement data for temporal modeling of pneumatic conveying process," in Proceedings of the Federated Conference on Computer Science and Information Systems (FedCSIS), pp. 283-286, IEEE, 2018, http:// ieeexplore.ieee.org/stamp/stamp.jsp?tp=\&arnumber $=8511266 \&$ isnumber $=8511145$.

[7] A. Romanowski, "Big Data-Driven Contextual Processing Methods for Electrical Capacitance Tomography," IEEE Transactions on Industrial Informatics, vol. 15, no. 3, Article ID 2855200, pp. 1609-1618, March 2019, http://ieeexplore.ieee.org/ stamp/stamp.jsp?tp=\&arnumber $=8410466 \&$ isnumber $=8661808$.

[8] T. Rymarczyk and G. Kłosowski, "Application of neural reconstruction of tomographic images in the problem of reliability of flood protection facilities," Eksploatacja i Niezawodność, vol. 20, no. 3, pp. 425-434, 2018.

[9] R. Sikora, Z. Giza, F. Filipowicz, and J. Sikora, "Bell function approximation of material coefficients distribution in the electrical impedance tomography," IEEE Transactions on Magnetics, vol. 36, no. 4 I, pp. 1023-1026, 2000.

[10] W. Smolik, J. Kryszyn, T. Olszewski, and R. Szabatin, "Methods of Small Capacitance Measurement in Electrical Capacitance Tomography," Informatics Control Measurement in Economy and Environment Protection, vol. 7, no. 1, pp. 105-110, 2017.

[11] M. Soleimani, C. N. Mitchell, R. Banasiak, R. Wajman, and A. Adler, "Four-Dimensional electrical capacitance tomography imaging using experimental data," Progress in Electromagnetics Research, vol. 90, pp. 171-186, 2009.

[12] M. Ziolkowski, S. Gratkowski, and A. R. Zywica, "Analytical and numerical models of the magnetoacoustic tomography with magnetic induction," COMPEL - The International Journal for Computation and Mathematics in Electrical and Electronic Engineering, vol. 37, no. 2, pp. 538-548, 2018.

[13] A. Zirak, P. Beygi, and S. Mirzakhah, "Dynamic estimation of the modeling error statistics in diffuse optical tomography," Inverse Problems in Science and Engineering, vol. 25, no. 4, pp. 492-505, 2017.
[14] A. R. Zirak and M. Khademi, "An efficient method for model refinement in diffuse optical tomography," Optics Communications, vol. 279, no. 2, pp. 273-284, 2007.

[15] P. Lopato, T. Chady, R. Sikora, S. Gratkowski, and M. Ziolkowski, "Full wave numerical modelling of terahertz systems for nondestructive evaluation of dielectric structures," COMPEL. The International Journal for Computation and Mathematics in Electrical and Electronic Engineering, vol. 32, no. 3, pp. 736-749, 2013.

[16] T. Rymarczyk, J. Sikora, K. Polakowski, and P. Adamkiewicz, "Effective ultrasound and radio tomography imaging algorithm for two-dimensional problems," Przeglad Elektrotechniczny, vol. 94, no. 6, pp. 62-69, 2018.

[17] S. Kaczmarz, "Angenäherte auflösung von systemen linearer gleichungen," Bull. Acad. Polon. Sci. Lett. A, vol. 6-8A, pp. 355357, 1937.

[18] A. C. Kak and M. Slaney, Principles of Computerized Tomographic Imaging, IEEE Press, New York, NY, USA, 1988.

[19] G. Kłosowski, A. Gola, and A. Świć, "Application of fuzzy logic controller for machine load balancing in discrete manufacturing system," in Proceedings of the International Conference on Intelligent Data Engineering and Automated Learning, pp. 256263, 2015.

[20] G. Klosowski, T. Rymarczyk, and A. Gola, "Increasing the reliability of flood embankments with neural imaging method," Applied Sciences, vol. 8, no. 9, p. 1457, 2018.

[21] E. Korzeniewska, I. Gałązka-Czarnecka, A. Czarnecki, A. Piekarska, and A. Krawczyk, "Influence of PEF on antocyjans in wine," Przegląd Elektrotechniczny, vol. 94, no. 1, pp. 57-60, 2018.

[22] A. Krawczyk and E. Korzeniewska, "Magnetophosphenes-history and contemporary implications," Przeglad Elektrotechniczny, vol. 1, no. 1, pp. 63-66, 2018.

[23] J. Kryszyn, W. T. Smolik, B. Radzik, T. Olszewski, and R. Szabatin, "Switchless charge-discharge circuit for electrical capacitance tomography," Measurement Science and Technology, vol. 25, no. 11, 2014.

[24] M. Majchrowicz, P. Kapusta, L. Jackowska-Strumillo, and D. Sankowski, "Acceleration of image reconstruction process in the electrical capacitance tomography $3 \mathrm{~d}$ in heterogeneous," Pomiary w Gospodarce i Ochronie Srodowiska (IAPGOS, vol. 7, no. 1, pp. 37-41, 2017.

[25] https://www.engineeringtoolbox.com/sound-speed-water-d_598 .html, [access: June 2018].

[26] https://en.wikipedia.org/wiki/Speed_of_sound, [access: June 2018].

[27] P. D. Barba, Multiobjective Shape Design in Electricity and Magnetism, vol. 47 of Lecture Notes in Electrical Engineering, Springer, 2010.

[28] http://www.mathworks.com/products/matlab/, [access: June 2018]. 


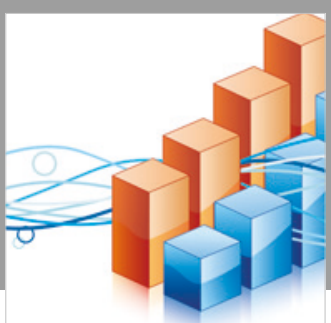

Advances in

Operations Research

\section{-n-m}
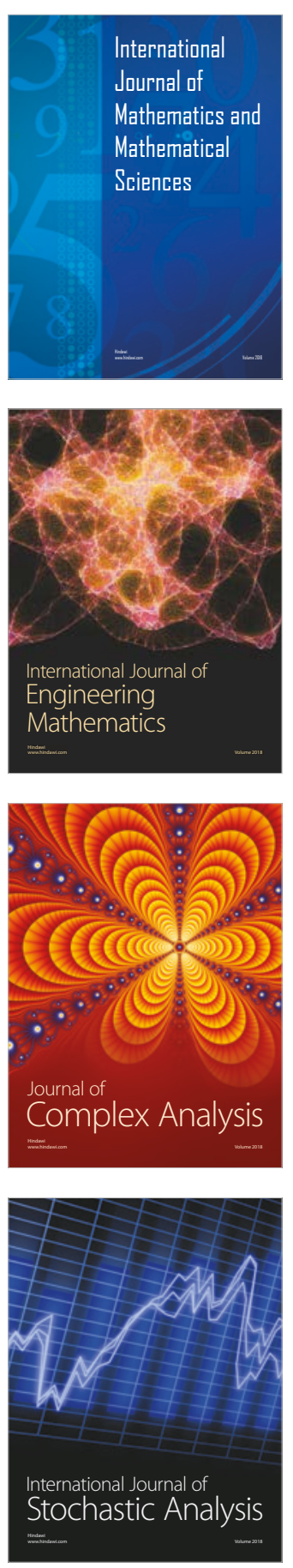
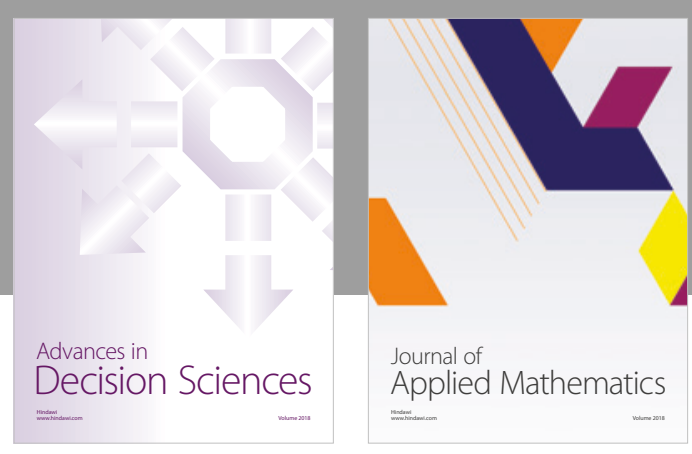

Journal of

Applied Mathematics
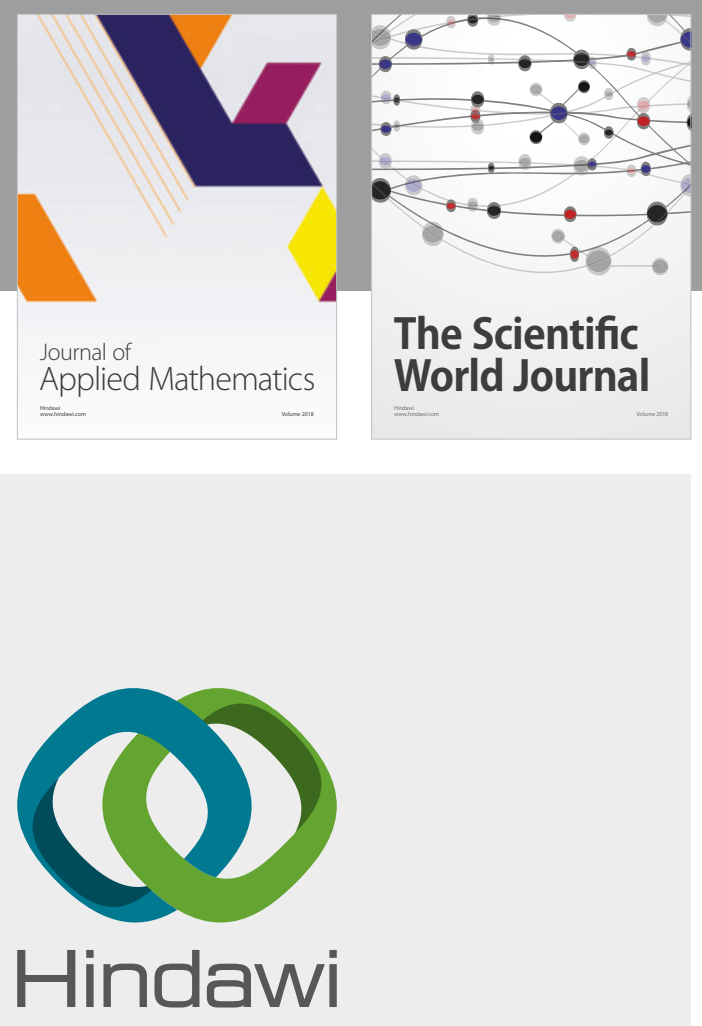

Submit your manuscripts at

www.hindawi.com

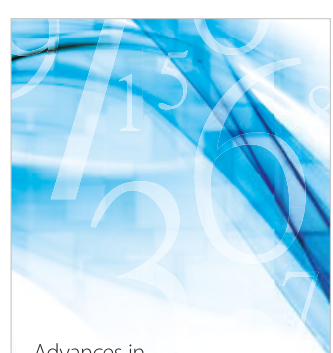

Advances in
Numerical Analysis
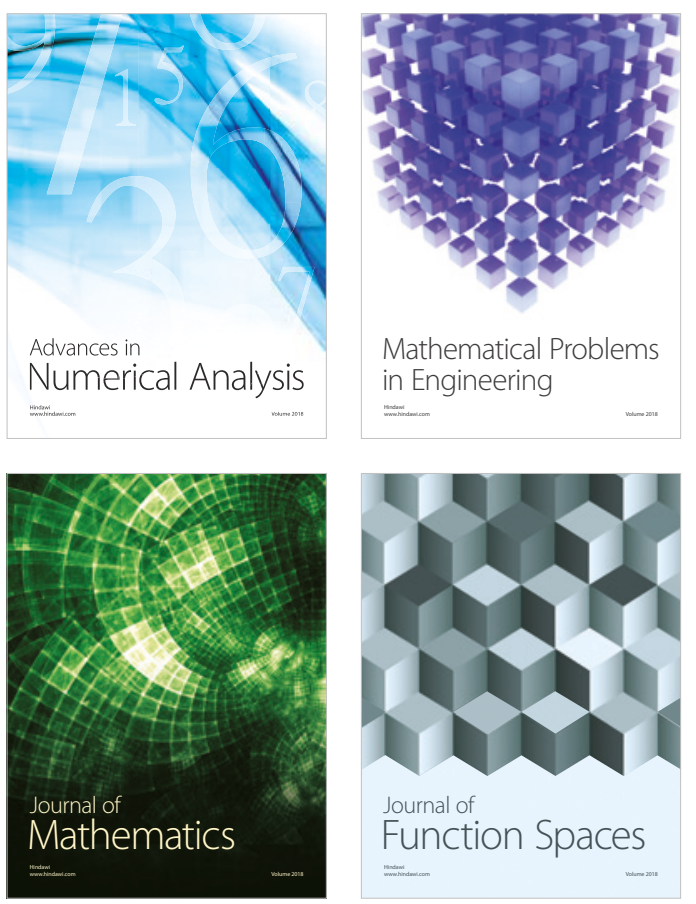

Mathematical Problems in Engineering

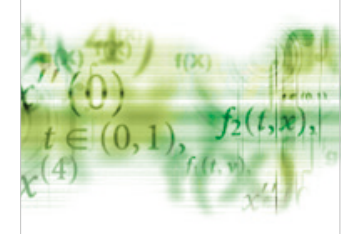

International Journal of

Differential Equations

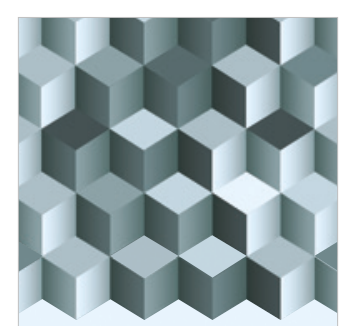

Journal of

Function Spaces

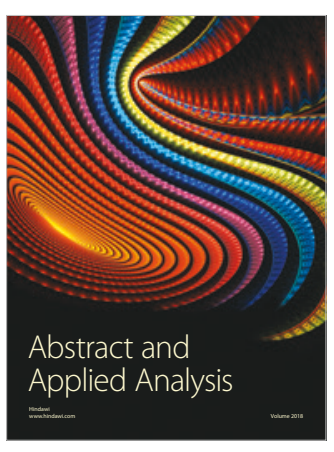

The Scientific

World Journal

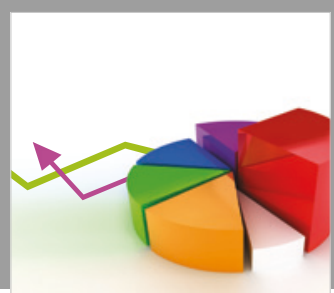

Journal of

Probability and Statistics
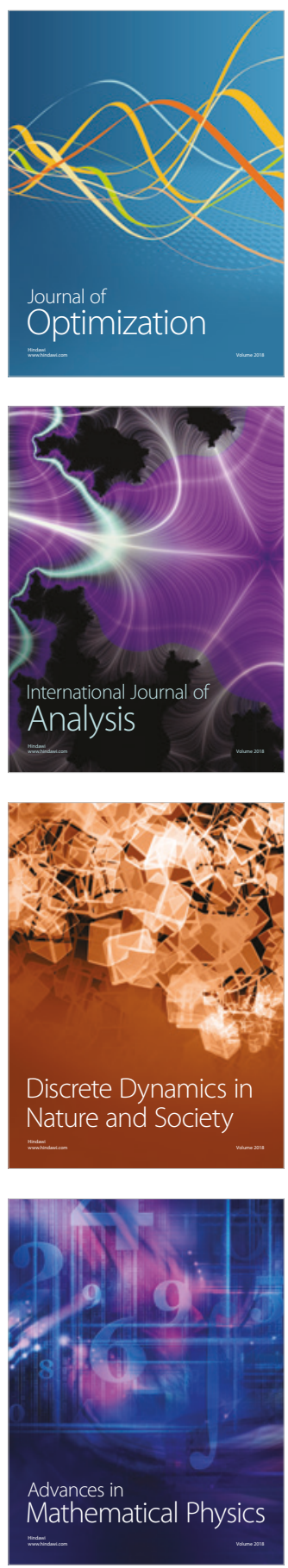